\title{
Alternative Ionization Methods for Particle Mass Spectrometry
}

\author{
William B. Whitten (PI) and Peter T. A. Reilly \\ Chemical and Analytical Sciences Division \\ Oak Ridge National Laboratory \\ Oak Ridge, Tennessee 37831-6142 \\ (865) 574-4921 FAX (865) 574-8363 whittenwb@ornl.gov
}

FY 2004 Annual Report to be submitted to the Environmental Management Science Program and to be posted on the internet.

\section{CAUTION}

The submitted manuscript has been authored by a contractor of the U.S. Government under contract no. DE-AC05-00OR22725. Accordingly, the U.S. Government retains a non-exclusive, royalty-free license to publish or reproduce the published form of this contribution, or allow others to do so, for the U.S. Government purposes.

Research at ORNL was sponsored by the Environmental Management Science Program of the Offices of Science and Environmental Management, U.S. Department of Energy, under contract no. DE-AC05-00OR22725 with Oak Ridge National Laboratory, managed and operated by UT-Battelle, LLC. 


\title{
Project 82792 FY 2004 Annual Report
}

\author{
Alternative Ionization Methods for Particle Mass Spectrometry
}

June 29, 2004

Principal Investigator: William B. Whitten, Oak Ridge National Laboratory, Bldg. 4500S, MS-6142, P.O. Box 2008, Oak Ridge, TN 37831-6142. Phone: 865-574-4921. FAX: 865-574-8363. E-mail: whittenwb@,ornl.gov

Co-Investigator: Peter T. A. Reilly, Oak Ridge National Laboratory, Bldg. 4500S, MS6142, P.O. Box 2008, Oak Ridge, TN 37831-6142. Phone: 865-574-4919. FAX: 865574-8363. E-mail: reillypt@ornl.gov

\section{Research Objective}

The objective of this project is to enhance the capabilities of a real-time airborne particle mass spectrometer by implementing matrix-independent methods for sample ionization. The enhancements should result in improved sensitivity for trace substances and, more importantly, permit quantitative determination of the presence of target species in microparticle samples on an individual particle basis.

We have used two different approaches to eliminate matrix effects on quantitative accuracy. Both rely on delayed ionization of ablated/desorbed neutral species so that ionization of target substances occurs after most of the expansion collisions have taken place. Resonance ionization by a tunable pulsed laser permits selective ionization of target species, with the laser tuned to a resonance transition from the ground state to an excited state of the target. Additional photons of the same (when possible) or different energy make up the energy required for ionization. The other approach is to perform the laser ablation step within a discharge so that desorbed neutrals are ionized by reactions with the plasma. Electron capture generates negative ions of substances with high electron affinity while electron impact ionization, associative ionization, and Penning ionization from excited metastable species produce positive ions in the discharge. Both atmospheric pressure Corona discharge ionization and glow discharge ionization at reduced pressure are being explored. Discharge ionization is relatively nonselective so that target specificity must be obtained through the mass spectral or MS/MS results.

Research Progress and Implications:This report summarizes work after year 3 of a 3year project. In prior years, we focused our efforts on two methods of discharge ionization: Corona discharge at atmospheric pressure external to the mass spectrometer, and glow discharge ionization with the discharge occurring in the moderate pressure region after the inlet orifice. An atmospheric pressure inlet system was constructed to guide ions formed outside the vacuum chamber into the ion trap for trapping and mass spectrometry. The ions were formed in a corona discharge between a high-voltage needle and the case. Particles were sampled within the discharge by a pulsed laser. Alternatively, vapor-phase samples could be ionized by collisions with atmospheric ions in the discharge (chemical ionization). In the past year, we have explored the possibility 
of altering the matrix itself instead of trying to modify the charge-transfer processes. This approach is the same that is used in MALDI, matrix-assisted laser desorption/ionization. In the usual implementation, molecules to be mass analyzed are dissolved in a matrix of molecules that are readily ionized by a pulsed laser. Charge exchange during the resulting expansion ionizes a portion

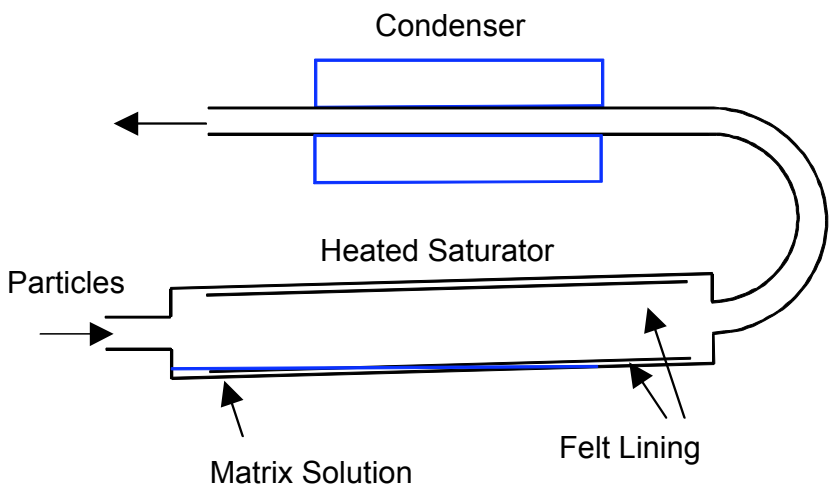

Fig. 1. Matrix applicator for coating single airborne particles for MALDI mass spectrometry of the analyte molecules. In our case, the species of interest are incorporated in an airborne particle that we want to analyze in real time. While we can not dissolve the analyte in a matrix, it is possible to deposit the matrix on the exterior of the particle by condensation while the particle is entrained in the entrance air stream of the apparatus. MALDI can then be performed if the laser pulse has sufficient fluence to desorb/ionize both a portion of the deposited matrix and particle. The matrix deposition makes use of condensation nucleation. The incoming particle, passing through a supersaturated vapor of the matrix, serves as a nucleus for condensation. As the coated particle is transported through the inlet to the vacuum system, residual matrix vapor is separated by differential

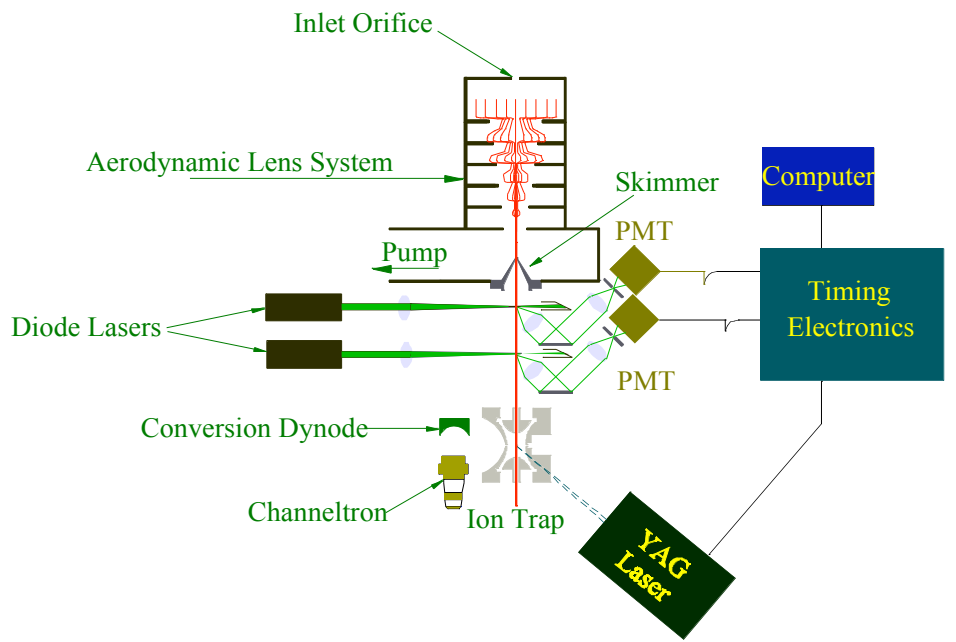

Fig. 2. Diagram of the single particle aerosol ion trap mass spectrometer. pumping.

Aerosols were generated with a Collison nebulizer, dried and coated with matrix in a heated saturator and cooled condenser, shown in Fig. 1. The heated saturator consisted of a tube (6" length, 3/4" diameter) lined with felt. Matrix solution was added and the tube was heated to $70 \mathrm{C}$. Picolinic acid, sinapinic acid, and 3-nitrobenzyl alcohol were used as matrices.

The temperature of the condenser was held between 3 and $10{ }^{\circ} \mathrm{C}$. The coated particles entered the instrument through an aerodynamic lens-based inlet that produces a collimated beam of particles $(>250-\square \mathrm{m}$ diameter) in the main chamber. Particles were sized by light scattering based time-of-flight. Typical particle diameters analyzed were 
between $400 \mathrm{~nm}$ and $1500 \mathrm{~nm}$. Next, the particles entered the ion trap where they were ablated and ionized on-the-fly with a $266-\mathrm{nm}$ laser $\left(4-8 \times 10^{6} \mathrm{~W} / \mathrm{cm}^{2}\right)$ when they reached the center of the trap. All aerosol experiments were performed in a Polaris Q ion trap mass spectrometer adapted to allow introduction and ablation/ionization of single particles in real rime. Ions above $1000 \mathrm{~m} / \mathrm{z}$ were detected through high mass resonance ion ejection. A diagram of the aerosol mass spectrometer is shown in Fig. 2.

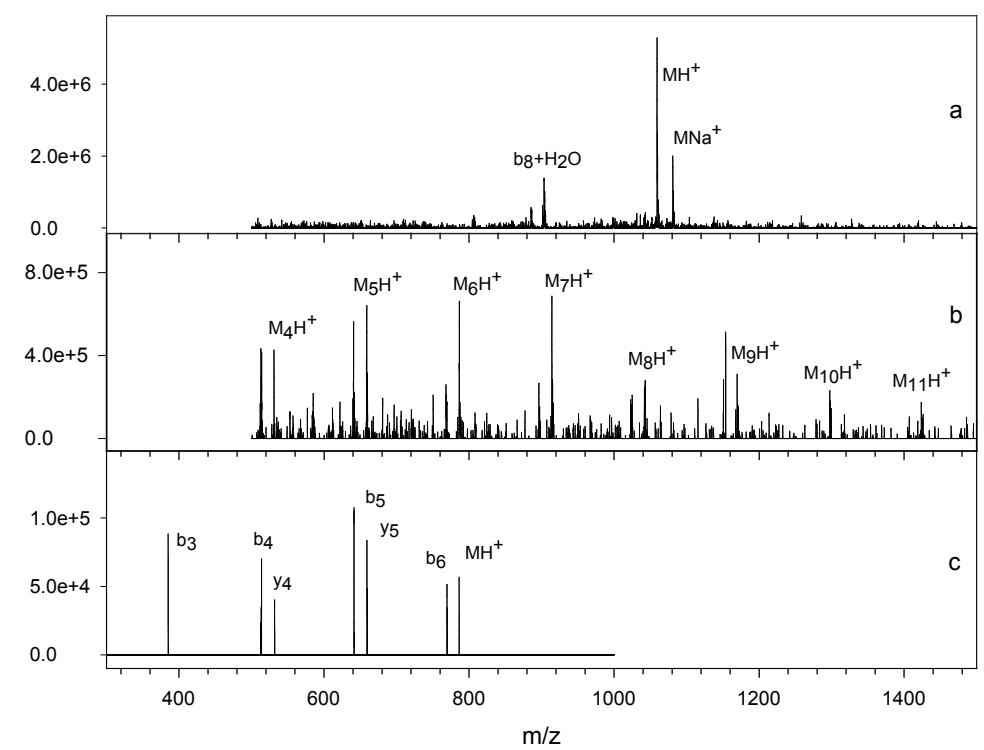

Fig. 3. Single particle MALDI mass spectra. a, bradykinin, $b$, polylysine, $c, M S / M S$ of a polylysine particle from $\mathrm{m} / \mathrm{z} 788$.
With the above approach, we were able to obtain single particle mass spectra of aerosols coated by the MALDI matrices mentioned above. Some examples of single particle mass spectra are shown in Fig. 3. Excellent sensitivity could be achieved. We estimate that for Fig. $3 \mathrm{~b}$, the particle contained 4 attomoles of the 11mer. We were able to obtain MS/MS spectra of single

particles as well, as shown in Fig. 3c. The ions at m/z 788 from a polylysine particle were isolated, dissociated by collisions with the buffer gas, and then mass analyzed, giving the resulting spectrum.

Single particle MALDI offers a means to detect substances that are difficult to ionize directly with the ablation laser. With this technique, we can extend our suite of detectable analytes to include substances such as aliphatic compounds, biomolecules, and PCBs. 